\title{
Refined Clothespin Relocation Test and Assessment of Motion
}

\section{Ali Hussaini 1 and Peter Kyberd 2}

I Institute of Biomedical Engineering, University of New Brunswick, Fredericton, NB, Canada

2 Department of Engineering Science, University of Greenwich, Kent, UK (formerly Institute of Biomedical Engineering, University of New Brunswick, Fredericton, NB, Canada)

\begin{abstract}
Background: Advancements in upper limb prosthesis design have focused on providing increased degrees of freedom for the end effector through multiple articulations of a prosthetic hand, wrist and elbow. Measuring improvement in patient function with these devices requires development of appropriate assessment tools.

Objectives: This study presents a refined clothespin relocation test for measuring performance and assessing compensatory motion between able-bodied subjects and subjects with upper limb impairments.

Study Design: Comparative analysis

Methods: Trunk and head motions of 13 able-bodied subjects who performed the refined clothespin relocation test were compared to the motion of a transradial prosthesis user with a single degree of freedom hand.

Results: There were observable differences between the prosthesis user and the able-bodied group. The assessment used provided a clear indication of the differences in motion through analysis of compensatory motion.

Conclusion: The refined clothespin relocation test provides additional benefits over the standard clothespin assessment and makes identification of compensatory motions easily identifiable to the researcher. While this paper establishes the method for the new assessment, further validation will need to be performed with more users.
\end{abstract}

\section{Clinical relevance}

The refined test provides a more defined structure for the trajectory of the hand/terminal device than the standard protocol for the clothespin relocation test. This will help researchers interested in motion studies of limb segments to efficiently compare and analyze motion between able-bodied and prosthesis user groups.

\section{Keywords}

Assessment, biomechanics, biomechanics of prosthetic/orthotic devices, motion analysis, outcome measure, prosthetics, rehabilitation, rehabilitation of prostheses users, transradial, upper limb prosthetics

Date received: 8 December 2015; accepted: 28 June 2016 


\section{Background}

Recent progress in assessment for upper limb prosthesis users has focused on measuring patient functionality. Improving functional outcomes with advanced prosthetic technology necessitates standardized testing to properly quantify and measure changes in the prosthesis user's utility with their assistive device. In the past, tools have been used to measure user ability, including motion capture $^{1-4}$, electro-goniometry ${ }^{5}$, visual attention ${ }^{6,7}$, task completion, and electromyography ${ }^{8,9}$. These methods indicate a particular aspect of the patient-prosthesis interaction and provide a sense of how activity occurs, but not a measure of how well the activity is performed. Motion capture to record limb motion or visual attention to measure cognitive effort, can enhance any measurement of the basic ability to complete the task. The method of measurement must be paired with the appropriate assessment (task to be performed) to form an index of patient functionality.

Defining the appropriate testing method is as important as the particular measurement tool used to measure patientprosthesis interaction. Outcome measures for many upper limb impairments ${ }^{10}$ and some specifically meant for those with upper limb loss" exist. The Upper Limb Prosthetic Outcome Measures (ULPOM) group is made up of researchers and therapists who are actively working to promote standardized outcome measures for prosthesis users ${ }^{12}$, to assess limitations, and to define a strategy for proper assessment of user abilities ${ }^{13}$. This will help to identify limitations in user motion and prosthesis function, and create a taxonomic wish list for prosthetics manufacturers designing advanced assistive devices. Having identified existing tools with sufficient psychometric properties, the next phase is to validate promising tools, or develop new ones that will fill gaps in the tool set ${ }^{14-16}$. This study aims to move the concept forward by developing a standardized assessment method that captures the motions and compensatory motions of able-bodied and prosthesis users, respectively.

In this study, the role of the wrist in able-bodied motion and the effect of wrist loss in a prosthesis user were investigated. The absence of the wrist severely limits optimal positioning of the hand for grasping and manipulation. Previous studies have investigated the restriction on range of motion the absence of the wrist creates and how a prosthesis user modifies the manner in which they use the rest of their arm to compensate ${ }^{17-19}$. These studies found that the inclusion of a prosthetic wrist affects kinematic motion of the user. The addition of the wrist allows kinematic motions that bear a greater resemblance to that of an able-bodied subject. A study by Kestner $^{20}$ found an increase in usage of a prosthetic wrist for certain activities (e.g. writing, eating). The survey by Atkins et al. ${ }^{21}$, indicated the need for wrist motion for survey respondents with limb absence of the upper extremity. Evidently, quantifying the effect of the wrist 
(loss and addition of) in a patient population provides insight into kinematic motion and improvement in patient function.

\section{Compensatory Motions, Repetitive Strain Injuries and}

\section{Overuse}

Compensatory motions are movements performed when a physical or neurological impairment changes the way a person can complete an action. For prosthesis users, the movements differ from the observable motion of an individual with a healthy limb. These motions accommodate for the decreased achievable range of motion or strength, the consequence of an amputation. The motions are made in an attempt to reduce the level of effort (i.e. the physical movement or mental load placed on the prosthesis user). For example, if an individual has a shoulder injury (e.g. impingement), they may reduce the motion of the shoulder and bend further at the waist to achieve an effective position for completing a task. For a given joint, the range of motion used in compensation by a prosthesis user tends to be much larger than that of an injured, but intact limb, as the complete absence of the joint eliminates the achievable positions for the adjacent limb segment, instead of simply reducing the range. Isolated instances of compensatory motion will generally not affect the user, but repeated use of compensations outside the typical range of the joint can increase stress on the muscle and joint and increase the potential for an over use injury, such as a repetitive strain injury $(\mathrm{RSI})^{22}$.
The overuse of compensatory movements on a user has not yet been measured effectively. There have recently been a handful of studies that focus on compensatory motions of upper limb prosthesis users ${ }^{1,3,23-26}$. The work by Zinck $^{24}$ showed that the same task may have different solutions paths. In addition, the definition of when a task is considered "complete" is important. This has to be further refined or else compensatory motion comparisons has a level of ambiguity introduced. The work by Carey et al.' found that users may compensate for limb loss with different motions and that these depend on the specific tasks they want to accomplish. An increase in range of motion of the torso may be a compensation for one activity, but another activity might require an increase in shoulder angle. This dependency on task is an important criterion that must be considered when an assessment is developed to evaluate the improvement in a new type of prosthesis and its effect on reducing RSIs. Unfortunately, the majority of studies on $\mathrm{RSI}^{27-30}$ have involved nonprosthesis users (e.g. baseball pitching). There has been little work so far in the effect of compensatory motions of prosthesis users and long term implications of RSI. A survey by Jones ${ }^{31}$ found that $50 \%$ of prosthesis user respondents developed problems in the intact limb. Some of these were due to overuse of the remaining limb, but other factors (arthritis, carpal tunnel, etc.) were also present. 
While it is possible to record bodily motions, it is more difficult to detect significant changes, and thus it is important to choose the correct test that is representative of user activities and can unambiguously illuminate compensations. After the tool for measurement of body motions has been selected, the assessment test must be chosen where wrist and hand control are necessary for task completion.

\section{Assessment - The Refined Clothespin Relocation Test (RCRT)}

The Rolyan Graded Pinch Exerciser was developed as a training tool for upper limb impairment. It was adapted for use as an assessment tool ${ }^{32}$. Three clothespins are moved from a horizontal rod onto a vertical rod (and vice versa), changing the orientation in the process. This motion is ideal in evaluating hand operation and independent wrist control, while making compensations in the trunk and shoulder obvious to the observer. Before implementation, the test had to be refined to control the arm's motions further.

Comparison of different motions is difficult if the motions are not constrained in some way. The study of walking through gait analysis works because the motions of an individual's strides are similar to the average of the population. Individual strides can be stretched or reduced temporally to match the general population using event markers such as heel strike or toe off. Use of different tools can constrain the motion of the upper limb to allow similar levels of analysis ${ }^{33,34}$ A constrained clothespin relocation test, designed to reduce the number of possible solution paths, offers another means to control the motion effectively.

\section{Method}

The refined protocol used in this study differs from the method used in previous work ${ }^{35}$, but provides an additional underlying structure to the trajectories the clothespins follow. When the trajectories are plotted over time, the modifications make compensatory motions more evident. The start and end positions, and order in which the clothespins are moved are now defined (Figure I). These changes make the comparison between individual trajectories and between the individual and the general population clearer.

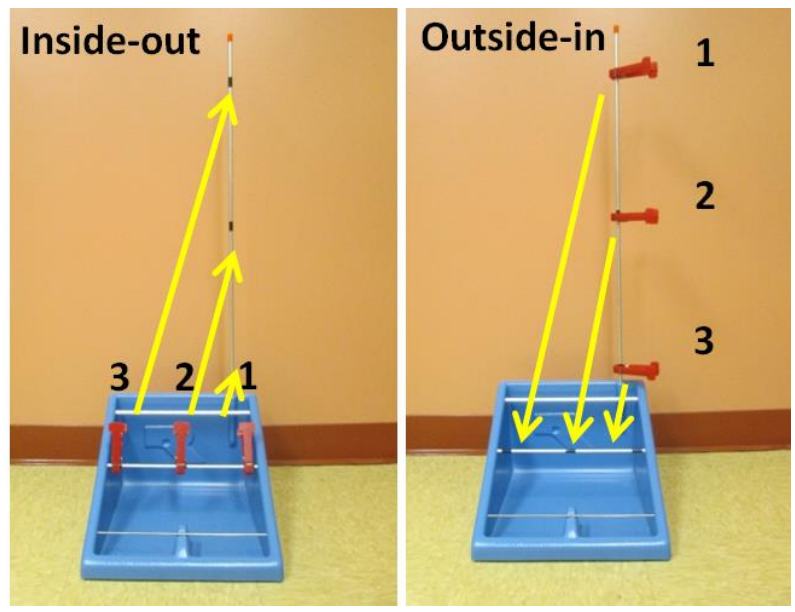

Figure I. Constrained assessment path for upward (left) and downward assessment (right) 
The particular order and directions were chosen to ensure ease of task completion by the prostheses users as the paths (arrows in Figure I) removed the need to place a prosthetic hand between two adjacent clothespins. This was a greater concern when moving the clothespins upward as there is a smaller distance between the clothespins (grasping the clothespin requires more fine motor control than placement).

When performing the test, the subjects stood at a table, adjusted to hip height, with the hand to be assessed by their side (opposite hand holding the base unit). A timer was placed on the same side as the hand under test and was started/stopped by the subject with that hand after three clothespins had been moved. The time was recorded. If a clothespin was dropped or feet left the floor, the timer was reset and the test was repeated. A cycle comprised of the clothespins being moved in both directions once. Five complete cycles were recorded during an assessment session, but only one upward and downward assessment was used for analysis. The chosen trial had the least amount of marker confusion and missing markers in capture volume. For more details on the complete procedure, see Hussaini ${ }^{23}$.

\section{Participants}

All I 3 able-bodied subjects were individuals with left-hand dominance. This ensured that their non-dominant hand was on the right side, matching the non-dominant hand of the single prosthesis user. The prosthesis was a two-site myoelectric prosthesis with a single degree of freedom hand with a flexion wrist (Motion Control Inc.), locked in the flexed position. The user was an experienced myoelectric prosthesis user (amputation due to trauma). He had been using myoelectric devices longer than 2 years and uses a myoelectric device at work, between 4-8 hours a day.

\begin{tabular}{|c|c|c|}
\hline Acronym & $\begin{array}{l}\text { Anatomical } \\
\text { Landmark }\end{array}$ & $\begin{array}{c}\text { Marker } \\
\text { Diameter } \\
(\mathrm{mm})\end{array}$ \\
\hline \multicolumn{3}{|c|}{ Head Markers } \\
\hline FRHD & Front of head, forehead & 25 \\
\hline LTHD & Left side of head & 25 \\
\hline RTHD & Right side of head & 25 \\
\hline C7 & C7 of the Spine & 25 \\
\hline LCLA & Left sternoclavicular joint & 19 \\
\hline RCLA & Right sternoclavicular joint & 19 \\
\hline \multicolumn{3}{|c|}{ Arm and Shoulder Markers } \\
\hline LSHO & $\begin{array}{l}\text { Acromion of Left side } \\
\text { (shoulder) }\end{array}$ & 25 \\
\hline LUPA & Upper Arm of Left side & 25 \\
\hline LELB & $\begin{array}{l}\text { Lateral epicondyle of Left } \\
\text { side }\end{array}$ & 25 \\
\hline $\mathrm{RSHO}$ & $\begin{array}{l}\text { Acromion of Right side } \\
\text { (shoulder) }\end{array}$ & 25 \\
\hline RUPA & Upper Arm of Right side & 25 \\
\hline RELB & $\begin{array}{l}\text { Lateral epicondyle of Right } \\
\text { side }\end{array}$ & 25 \\
\hline \multicolumn{3}{|c|}{ Forearm Markers } \\
\hline LRAD & Left radial styloid process & 19 \\
\hline LULNA & Left ulnar styloid process & 19 \\
\hline LWC & Left wrist centre & 19 \\
\hline RRAD & Right radial styloid process & 19 \\
\hline RULNA & Right ulnar styloid process & 19 \\
\hline RWC & Right wrist centre & 19 \\
\hline \multicolumn{3}{|l|}{ Hand Markers } \\
\hline L2MC & $\begin{array}{l}\text { Left } 2 \text { nd metacarpal head, } \\
\text { below knuckle }\end{array}$ & 19 \\
\hline L5MC & Left 5 th metacarpal head & 19 \\
\hline R2MC & 2nd metacarpal head & 19 \\
\hline R5MC & 5th metacarpal head & 19 \\
\hline RIFIN & $\begin{array}{l}\text { Right Index finger, distal to } \\
\text { DIP joint }\end{array}$ & 13 \\
\hline \multicolumn{3}{|l|}{ Hip Markers } \\
\hline SACR & Flat part of sacrum & 25 \\
\hline LHIP & $\begin{array}{l}\text { Left front of ASIS, } \\
\text { extended on wand }\end{array}$ & 25 \\
\hline RHIP & $\begin{array}{l}\text { Right front of ASIS, } \\
\text { extended on wand } \\
\text { Table I Subject Marke }\end{array}$ & 25 \\
\hline
\end{tabular}




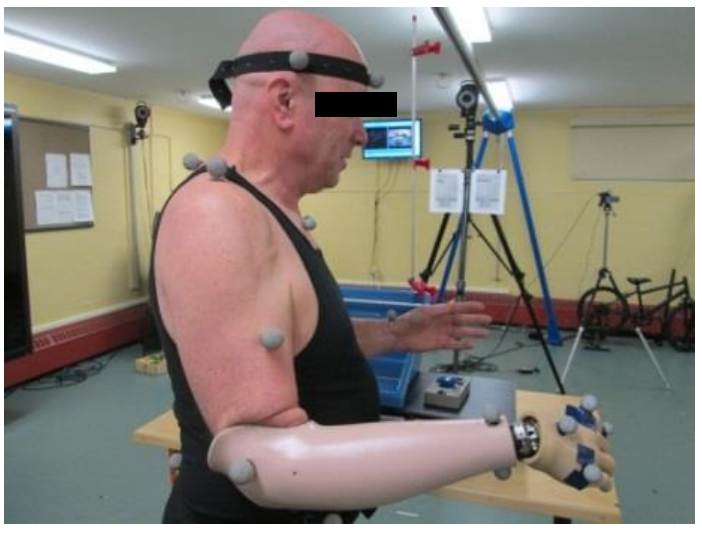

Figure 2. Reflective markers on prosthesis, sagittal view

This study received ethical approval from the UNB Research Ethics Board (REB 20|3-I|4). All subjects provided written informed consent for participation.

\section{Motion Capture}

Motion capture sessions were performed using an 8 camera Vicon M-Cam system [Oxford Metrics, Oxford, England] which tracked the positions of reflective spherical markers attached to the subjects. Trials were also recorded with a synched video camera. 26 spherical reflective markers were placed on boney landmarks on each subject (Table I). The placement of markers is shown in Figure 2.

The motion data was processed in the Vicon Workstation software to ensure complete trajectories and fill any gaps that result from marker occlusions. Marker trajectories were filtered with a Zero lag 4th-order Butterworth filter with a $5 \mathrm{~Hz}$ cutoff frequency.

\section{Data Analysis}

Local coordinate systems were defined for the pelvis, torso, and head and relative motion angles between these were calculated. A detailed discussion of how local coordinates systems were defined can be found in the works of MacPhee ${ }^{18}$ and Zinck ${ }^{24}$. Table 2 list the 8 motion angles that were analysed, though only the ones showing clear differences are plotted below.

\begin{tabular}{ll} 
Rotation & $\begin{array}{l}\text { Positive } \\
\text { Direction }\end{array}$ \\
\hline Trunk Relative to Pelvis & \\
\hline $\begin{array}{l}\text { Lateral Tilt } \\
\text { Flexion and Extension } \\
\text { Rotation }\end{array}$ & $\begin{array}{l}\text { Dominant side } \\
\text { Trunk forward } \\
\text { Dominant side }\end{array}$ \\
\hline Head Relative to Trunk & \\
\hline Lateral Tilt & Dominant side \\
Flexion and Extension & Head forward \\
Rotation & Dominant side \\
\hline Arm Angles & \\
\hline Shoulder Angle & Flexion / Abduction \\
Elbow Flexion and Extension & Flexion
\end{tabular}

Table 2 Motion angles and positive direction

\section{Results}

Figure 3 (upwards) and Figure 4 (downwards) display the motion angles of the prosthesis user, and the able-bodied subjects whom are represented by a shaded boundary curve. This represents a $95 \%$ confidence interval with the standard deviation from the arithmetic mean of the ablebodied subjects marking the upper and lower limits of the confidence interval. The prosthesis user is shown in the solid black line. The horizontal axis is normalized between $0 \%$ (start task) and 100\% (end task), when the subject 
pushes the timer button. The trajectories are fit to a cubic spline with 400 data points.

The prosthesis user's trajectory shows three distinct peaks which correspond to placement of the three clothespins onto the vertical rod. The magnitude peaks are indicative of the distance between start and end location of each clothespin, and the defined order of movement ensures that any segment of the trajectory can be easily attributed to a specific clothespin.

In the upward assessment, the lateral tilt trajectories had a range of motion that did not exceed 15 degrees (the prosthesis user). By analyzing the prosthesis user's trajectory, the periods in which the clothespins were placed onto the vertical bar can be identified, as lateral tilt (and trunk flexion) was required during this period. The initial large trough (centred about 25\%), corresponds to the first clothespin being placed. This resulted in the largest lateral tilt angle towards the non-dominant side as the user was unable to rotate the hand to a position where they could place the clothespin on the lowest position of the vertical rod. The user then returned to neutral (trunk angle) to grasp the second clothespin. Placement of the second clothespin occurred at the minimum (60\%), which is seen in the trajectory. The higher the clothespin needed to be placed, the less lateral tilt was displayed by the prosthesis user.
Head lateral tilt had a larger range of motion than the trunk as it was the motion that contributed the most in keeping the eyes focused on the task (more than the trunk).

Trunk and head flexion both produced trajectories that prominently showed the points at which the clothespins were placed on the vertical rod. The negative trajectories of the graph at the three clothespin locations corresponds to the point of placement onto the vertical rod, which required that the subject lean back when lifting the arm upwards. The positive return to a neutral position on the plot corresponds to the point after the clothespin had been placed and the user bent forward to grasp the next clothespin. It is apparent in the trajectory that the prosthesis user experienced a greater range of trunk flexion motion for each clothespin placement.

Head flexion was linked to the motion of the trunk, but had the larger range of motion. This was expected as the subjects appear to look down towards the table to grasp, and upwards to place the final clothespin. All subjects had a head flexion range of motion that exceeded 25 degrees. All trajectories began as positive (head flexed forward), to pick up the first clothespin and then became progressively more negative (head flexed backward) when placing each clothespin on a higher position than the previous.

Trunk rotation of the prosthesis user has the largest difference compared to the able-bodied group when 
placing the third clothespin. This resulted in more than 25 degrees of trunk rotation to the dominant side.

Head rotation remained negative for most of the task for all subjects. The vertical rod was on the right side of the subject which resulted in a negative rotation of the head when placing. Positive angles were measured when the subjects returned to the horizontal rod to grasp a clothespin, with the final clothespin producing the least negative or positive angle for the subjects. This was a result of the third clothespin being to the left (positive side) of the midline.

In the downward trajectory, the maximum range of motion occurred during manipulation of the first clothespin with a lateral tilt to the subjects' dominant side to get the hand up to the top clothespin. A large negative spike can be seen at $28 \%$. This was not due to excessive tilt to the right side, but the result of the right clavicle marker disappearing from view, likely due to the humerus and prosthetic hand obstructing the view of 3 of the cameras.

Trunk flexion and rotation, like trunk lateral tilt, mimics the upward assessment in that the largest flexion angle occurred when grasping the clothespin at the top location and then proceeded to reduce as the clothespins were grasped at a lower height. This is true for both able-bodied and prosthesis user, though the range is larger with the $\mathrm{pr}$
Figure 5 displays the completion times for the 5 trials of all the subjects in the upward direction. The prosthesis user is also shown (plus sign). There was an improvement (decrease) in time from the first to last trial in both the able-bodied subjects and the user. In the fourth trial, the prosthesis user had a slight increase in time to complete, due to a delay in stopping the timer after the clothespins had been successfully moved. The mean time to complete the trials for the able-bodied group was 5.46 seconds. The user took twice as long to complete. If only the final trial is considered (acclimation to the test), the user took 2.3 times as long as the mean of all the subjects.

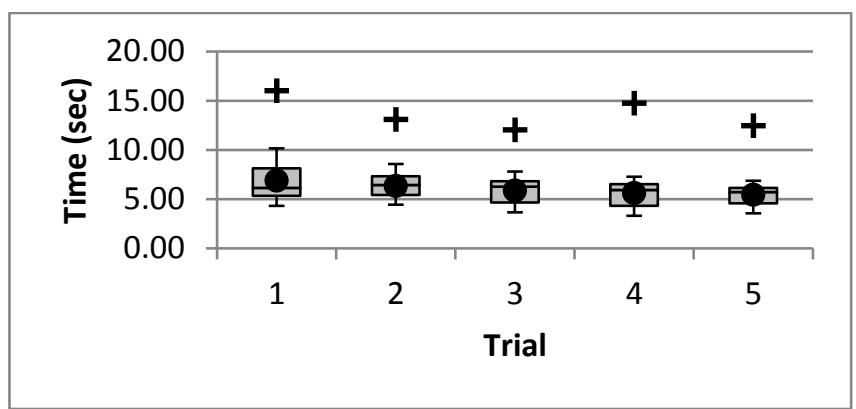

Figure 5 Clothespin upward assessment times for ablebodied subjects and prosthesis user $(+)$

The downward test (not shown) had similar results in terms of a decreasing time to complete across the trials.

The mean time for the able-bodied subjects was 5.42 seconds. Comparing the upward and downward trials, the able-bodied users had the largest difference in the second trial ( 0.23 seconds), but this was inconsequential. The prosthesis user took 3.97 seconds longer to complete the final downward trial, compared to the final upward trial. This suggests that the downward trial may be more 
difficult when there is a restriction on achievable motions for the hand.

\section{Discussion}

The RCRT lends itself well to analysis between subjects and between able-bodied subjects and the prosthesis user. Compared to other upper limb assessments, the shorter length of capture, the ordered trajectory and the standing position provided a more standardized testing. In using the RCRT, deviations from the motions of the able-bodied subjects were made more apparent in the trunk and head motions.

Trajectories for the left arm were not shown as minimal motion occurred in these joints. The decision to keep the left hand in the same position throughout each test was effective at producing compensatory motions in the trunk, head, and right arm. It also reduced the chance of the left hip marker being blocked from view of the cameras.

The ULPOM group had identified that although there are measurement tools that assess performance of the upper limb and those that measure user control, variations in protocol at the research institute level and validation of assessments tools with patient populations other than prosthesis users continues to make it difficult to compare and pool results. This is unfortunate as upper limb prosthesis users already represent a small patient population and larger data would result in easier validation of an assessment tool ${ }^{12}$. The RCRT modifies the original clothespin test to ensure that users perform the same test and perform it the same way. In this study, all the subjects were left-hand dominant but it would not be difficult to place a second vertical rod onto the left hand side of the Pinch Exerciser and have right-hand dominant users perform the same test with their left hand. This would also allow for users that wear their prosthesis on their left side to be added to this experimental population. The RCRT also removes the researcher from the test, in that the test is self-timed by the subject and can be administered (repeatable) by any other researcher. This addresses the issues raised by the ULPOM group regarding test validity and reliability, identified as key aspects for any outcome measure ${ }^{12}$. Future studies should include inter-rater reliability testing across multiple research centres. The test should also be performed in prosthetic clinics, though alternatives to expensive motion capture technologies may have to be explored.

Through observation of the compensatory motion and decreasing time to complete throughout the five trials, a priority structure may exist in how a prosthesis user approaches a task, especially one they have to repeat. In the first trial (upward or downward), the primary focus seemed to be on completing the task, with subsequent trials being about speed and developing a sense of confidence in the device. Accuracy, speed, compensation seemed to be the general progression. 
When a new prosthetic intervention is employed (e.g. multi-articulated hand, powered wrist), the time needed to switch to and utilize a different aspect of the terminal device becomes important, and provides insight to the cognitive effort and ease of control the addition provides. For example, when performing the RCRT, if a new prosthetic device has a powered wrist flexor, but controlling it requires a great deal of effort, the user might still opt to not use the flexor, but over-rotate their shoulder to position the hand to complete the task. The time taken would be low, but the compensatory motions would indicate whether the device is useful. However, if the control is improved, both the time to complete and compensatory motion angles would decrease, providing a measure of improvement in overall patient functionality.

While this study only included one prosthesis user, full validation will require extensive testing to establish the psychometric properties of the tool ${ }^{10}$. This study represents the first stage; establishing the method and recording the able bodied population dynamics. The next stage is to perform the test with multiple users, with different levels of limb loss, utilizing various terminal devices to perform the RCRT.

\section{Conclusion}

The RCRT required movement in all axes to accomplish the task of moving clothespins effectively. In particular, the ability to control the wrist can improve performance or influence kinematics on this test, and excessive motion of a body segment are easily identified. In order to compare subjects and to avoid comparisons that are simply qualitative observations, it was important that constraints and restrictions were placed on how a task was to be completed. Previous research has noted that subjects compensate for different tasks with different motions. Subjects will also complete the same task in different ways, which illustrates the importance of further defining and standardizing the idea of task completion. The particular protocol used in this study (in which the start and end location for the clothespins were ordered and constrained), allowed for a standardization of the trajectory paths to completing a task, which will make comparison between different subjects and prosthesis users more substantial.

\section{Acknowledgements}

The authors would like to thank the clinical team at the Atlantic Clinic for Upper Limb Prosthetics in Fredericton for coordinating patient visits for this study. The authors would also like to acknowledge the assistance of Dr Chris McGibbon at the University of New Brunswick for his direction with normalization of the trajectory data.

\section{References}

[I] Carey SL, Highsmith MJ, Maitland ME et al. Compensatory movements of transradial prosthesis users during common tasks. Clin Biomech (Bristol, Avon) 2008; 23:1 I 28-35.

[2] Bouwsema $H$, van der Sluis CK, and Bongers RM. Movement characteristics of upper extremity prostheses 
during basic goal-directed tasks. Clin Biomech 2010; 25(6): 523-529.

[3] Metzger AJ, Dromerick AW, Holley RJ et al. Characterization of compensatory trunk movements during prosthetic upper limb reaching tasks. Arch Phys Med Rehabil 2012;

[4] Hebert IS, and Lewicke I. Case report of modified Box and Blocks test with motion capture to measure prosthetic function. J Rehabil Res Dev 2012 49(8): I | 63-74.

[5] Hargrove, Ll, et al. Toward the development of a neural interface for lower limb prosthesis control. In Engineering in Medicine and Biology Society, EMBS 2009 annual international conference of the IEEE, Minneapolis, 2-6 September. IEEE, 2009.

[6] Bouwsema $\mathrm{H}$, et al. Determining skill level in myoelectric prosthesis use with multiple outcome measures." J Rehabil Res Dev 20 I2; 49(9): |33|-I348.

[7] Sobuh, MMD, et al. "Visuomotor behaviours when using a myoelectric prosthesis. J NeuroEngineering Rehabil 20।4; I I(72): I-II.

[81 Morita, S, Toshiyuki K, and Koii Ito. Estimation of forearm movement from EMG signal and application to prosthetic hand control. In Robotics and Automation, 2001. ICRA, Seoul, 2I-26 May. IEEE, 200 I.

[91 Scheme E, et al. Motion normalized proportional control for improved pattern recognition-based myoelectric control. Neural Systems and Rehabilitation Engineering, IEEE Transactions on 20I4; 22(I): I49-I57.

[101 Wright V. Prosthetic outcome measures for use with upper limb amputees: a systematic review of the peerreviewed literature, 1970 to 2009. J Prosthet Orthot 2009; $21(9): 3-63$.

[II] Miller LA and Swanson S. Summary and recommendations of the academy's state of the science conference on upper limb prosthetic outcome measures. J Prosthet Orthot 2009; 83-89.

[12] Hill W, et al. Upper Limb Prosthetic Outcome Measures (ULPOM): a working group and their findings. J Prosthet Orthot 2009; 21(9): 69-82.

[I3] Hill W, et al. Functional outcomes in the WHO-ICF model: establishment of the Upper Limb Prosthetic Outcome Measures Group. J Prosthet Orthot 2009; 21 (2): II5-II9.

[14] Miller et al. "Validation of four outcome measures for upper limb prosthesis use assessment." In Proceedings of ISPO World Congress 2015, Lyon, France, 22-25 June 2015, PP 442
[15] Burger et al. "Development of the Orthotic Prosthetic Users' Survey - Upper Extremity Functional Status module version 3.0 - OPUS-UEFS 3.0." In Proceedings of ISPO World Congress 2015, Lyon, France, 2225 June 2015, pp 289

[16] Hermansson et al. "Development of the Assisting Hand Assessment-PAD: A Rasch-built performance measure for people with unilateral upper limb prosthesis, amputation or reduction deficiency." In Proceedings of ISPO World Congress 2015, Lyon, France, 22-25 June 2015, PP 570

[17] Ross M. Development of a Quantitative Test for Prosthetic Function Using Motion Analysis and Activities of Daily Living. Master's Thesis, University of New Brunswick, 2005. pp.40-4I.

[18] MacPhee B. Examining the Prosthetic Function and Body Behaviour of Prosthesis Users Performing Activities of Daily Living. Master's Thesis, University of New Brunswick, 2007. pp.68-74.

[19] Bertels et al. Objectifying the functional advantage of prosthetic wrist flexion. J Prosthet Orthot 2009; 2 I (2); 7478.

[20] Kestner S. Defining the relationship between prosthetic wrist function and its use in performing work tasks and activities of daily living.J Prosthet Orthot 2006; 18(3): 80-86.

[21] Atkins D], DCY Heard, and Donovan WH. Epidemiologic Overview of Individuals with Upper-Limb Loss and Their Reported Research Priorities. J Prosthet Orthot I 996 8(I): 2-II.

[22] Kidd PS, McCoy C, and Steenbergen L. Repetitive Strain Injuries in Youths. J Am Acad Nurse Pract 2000; 12(10): 413-426.

[23] Hussaini A. Independent Pro-supination Control in Transradial Myoelectric Prosthesis Users. Master's Thesis, University of New Brunswick, 2015

[24] Zinck A. Investigation of Compensatory Movements in Prosthesis Users and the Design of a Novel wrist. Master's Thesis, University of New Brunswick, 2008. pp.22-23

[25] Major M], et al. Comparison of range-of-motion and variability in upper body movements between transradial prosthesis users and able-bodied controls when executing goal-oriented tasks. J NeuroEng Rehabil 20 I4; I I(I).

[26] Carey SL, et al. Kinematic comparison of myoelectric and body powered prostheses while performing common activities. Prosthet Orthot Int 2009; 33(2): I79-I86. 
[27] Gambrell CR. Overuse syndrome and the unilateral upper limb amputee: consequences and prevention. J Prosthet Orthot 2008; 20(3): I26-I 32.

[28] Fortenbaugh D, Fleisig GS, Andrews JR. Baseball pitching biomechanics in relation to injury risk and performance. Sports Health 2009; I:3 |4-320.

[29] Hansson GA, et al. Physical workload in various types of work: Part I. Wrist and forearm. Int. J. Ind. Ergon 2009; 39:22I-233

[30] Muggleton JM, Allen R, Chappell PH. Hand and arm injuries associated with repetitive manual work in industry: a review of disorders, risk factors and preventive measures. Ergon 1999; 42(5): 7|4-739.

[3I] Jones LE, Davidson JH. Save that arm: a study of problems in the remaining arm of unilateral upper limb amputees. Prosthet Orthot Int 1999; 23(I): 55-58.
[32] Kuiken, TA et al. The use of targeted muscle reinnervation for improved myoelectric prosthesis control in a bilateral shoulder disarticulation amputee. Prosthet Orthot Int 2004; 28(3): 245-253.

[33] Murgia et al. Marker placement to describe the wrist movements during activities of daily living in a cyclical task. Clin Biomech 2004; 19(3): 248-254.

[34] Murgia A, Kyberd PJ, Barnhill T. The use of kinematic and parametric information to highlight lack of movement and compensation in the upper extremities during activities of daily living. Gait Posture. 2010; 3 I (3): 300-306.

[35] Lipschutz RD, et al. Shoulder disarticulation externally powered prosthetic fitting following targeted muscle reinnervation for improved myoelectric control. J Prosthet Orthot 2006; 18(2): 28-34. 


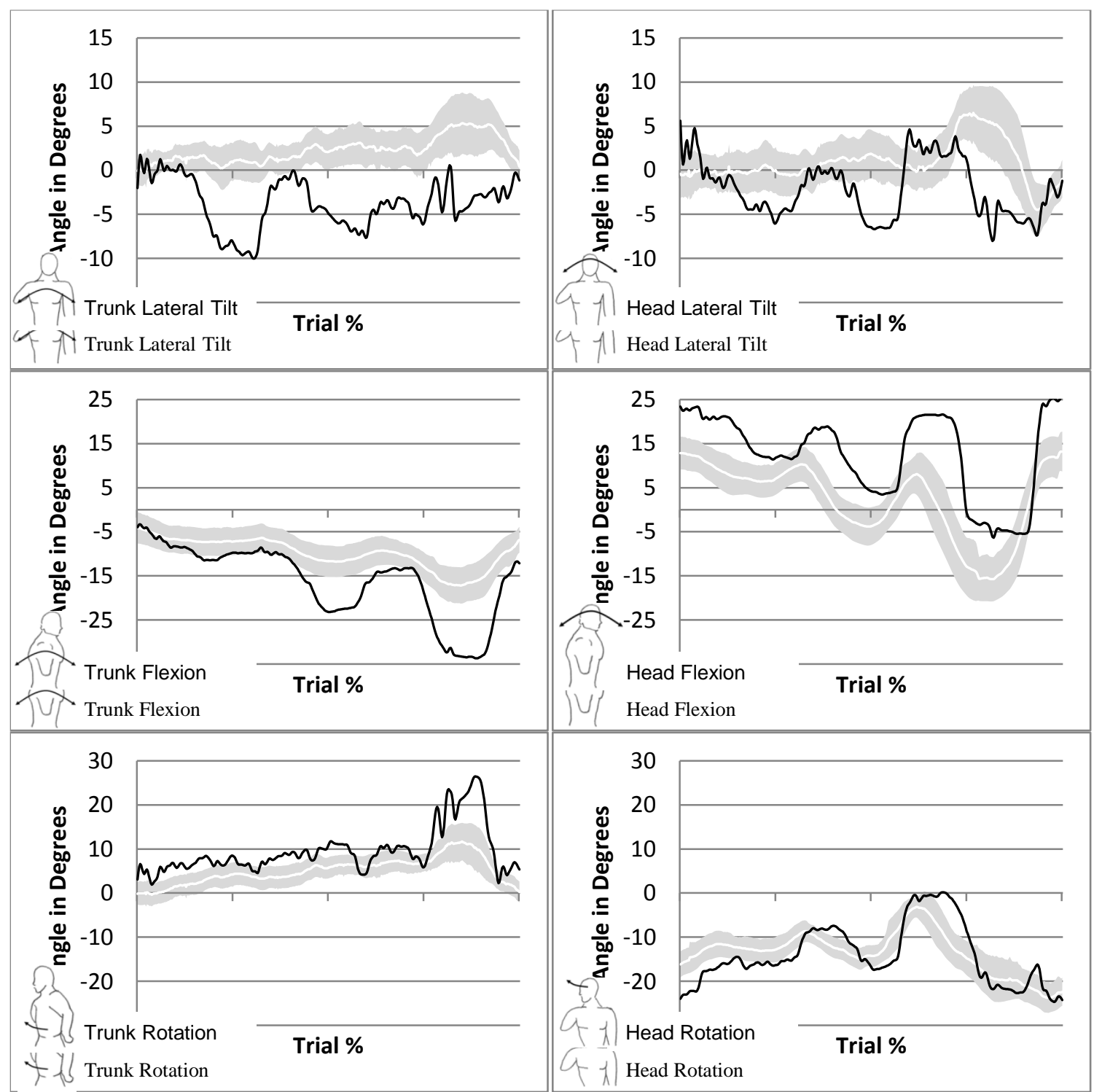

Figure 3. Confidence bound (95\%) for trajectories, clothespin upward test. Trajectories for the prosthesis user are shown in black, with able-bodied subjects represented by the shaded boundary region. Trunk motions are displayed on the left with the corresponding motions for the head on the right side. 


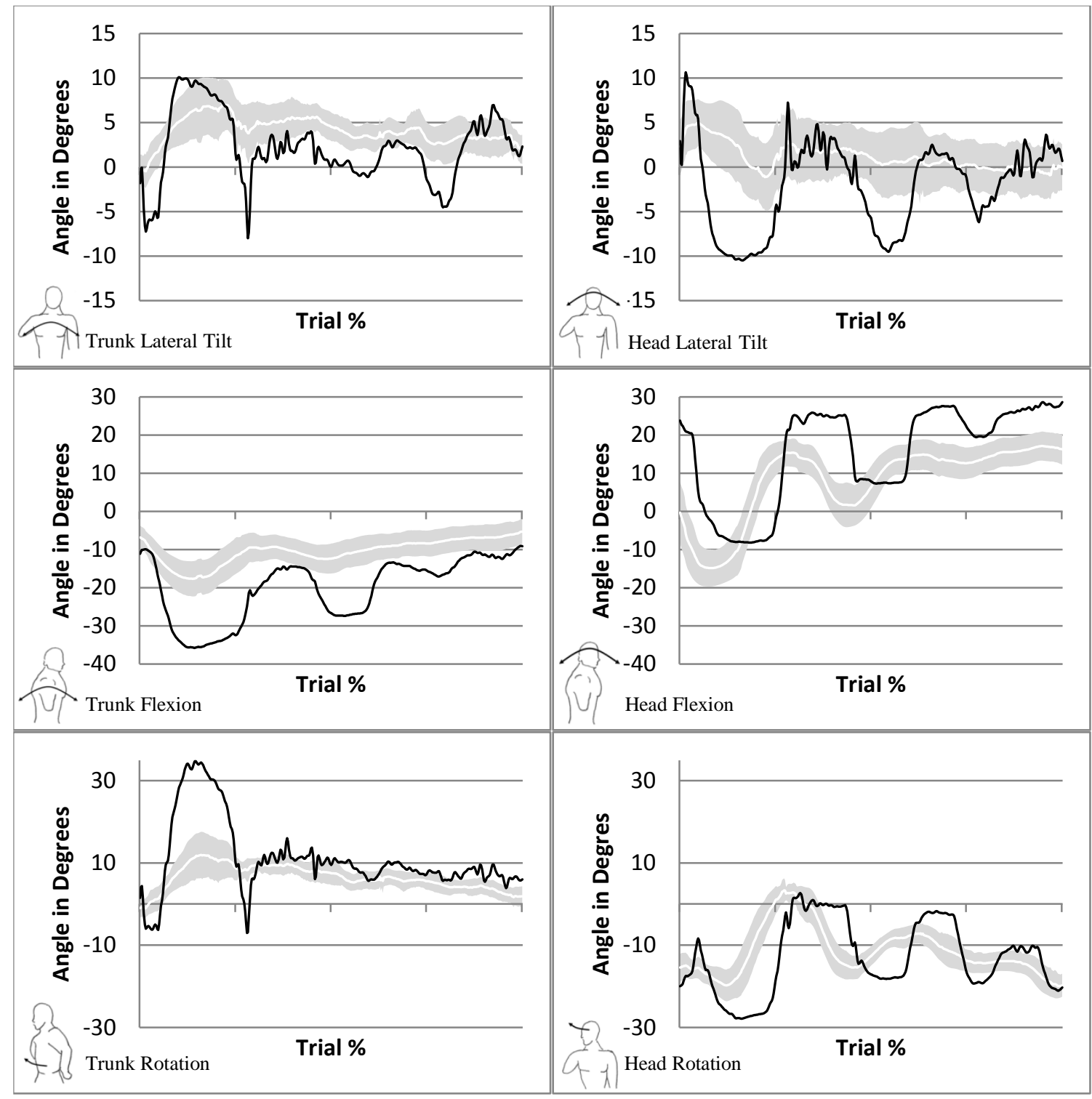

Figure 4. Confidence bound (95\%) for trajectories, clothespin downward test. Trajectories for the prosthesis user are shown in black, with able-bodied subjects represented by the shaded boundary region. Trunk motions are displayed on the left with the corresponding motions for the head on the right side. 